Sādhanā Vol. 39, Part 4, August 2014, pp. 809-818. (C) Indian Academy of Sciences

\title{
Developing an intelligent control system of automatic window motor with diverse wireless sensor network devices
}

\author{
YAO-CHIANG KAN ${ }^{1}$, HSUEH-CHUN LIN ${ }^{2}$ and \\ WEN-PEI SUNG ${ }^{3, *}$ \\ ${ }^{1}$ Department of Communications Engineering, Yuan Ze University, \\ No. 135 Yuan-Tung Road, Chung-Li, Taoyuan 32003, Taiwan \\ ${ }^{2}$ Department of Health Risk Management, China Medical University, \\ No. 91 Hsueh-Shi Road, Taichung 40402, Taiwan \\ ${ }^{3}$ Department of Landscape Architecture, National Chin-Yi University of Technology, \\ No.57, Chung Shan Road, Sect. II, Taiping, Taichung 41170, Taiwan \\ e-mail:wps@ncut.edu.tw
}

MS received 21 July 2013; revised 19 May 2014;accepted 24 May 2014

\begin{abstract}
This invention system involves hardware, firmware and software to develop an intelligent control system of automatic window motor with diverse wireless sensor network (WSN) devices for health and environmental monitoring. The parts of this invention are improved by implementing the WSN mote into environmental sensors that may detect temperature, humility, toxic gas, smog or aerosol, etc. With embedded system design, these sensors are capable of delivering WSN signal packets based on ZigBee protocol that follows the IEEE 802.14.4 standards. The primary hardware of the system is the window motor with circuit design by integrating micro control unit (MCU), radio frequency (RF) and WSN antenna to receive command. The firmware developed under embedded system can bridge hardware and software to control the window at the specified position. At the back end, the control system software can manage diverse sensor data and provide the interface for remote monitoring.
\end{abstract}

Keywords. Intelligent control; automatic window; wireless sensor network (WSN); micro control unit (MCU).

\section{Introduction}

Incorporating various advanced energy-related technology in the design and construction of new buildings will greatly raise the value of the building. Existing old buildings may achieve great reduction of energy consumption by improving management and adding relevant energy-saving equipment. The windows may be subjected to drastic renovation to satisfy the requirement for

*For correspondence 
lowering the energy consumption while maintaining a comfort building; a set of well-designed windows may lead to significant profits due to energy saving. Conversely, poorly designed windows will cause heavy burdens on the building energy consumption. Taiwan is located in sub-tropical region with unique hot and humid environment. Hence, how to achieve exchange of indoor and outside air to improve the energy efficiency without sacrificing comfortable indoor living environment is becoming an important research topic.

The window curtain and window glass influence the design and function of a window to a great extent. Installing an adequate window curtain will keep the room warm in winter and cool in summer. The heat loss of a tightly fit window curtain will be more effective than the curtain made of dense and heavy material but loosely hung in maintaining a comfort indoor environment in winter. In summer, the window curtain will lower the quantity of heat transmitted into the room. The effectiveness of the window curtain in reducing heat transmission through window is affected by three factors, i.e., solar energy radiation reaching the window glass, the capacity of window curtain to absorb heat, and the quantity of solar energy penetrate the window glass and curtain. Except the new Low-E (Low-emissivity glass) glass, current window glass may include clear glass, heat-absorbing (dyed) glass and heat-reflecting glass. The clear glass generally has the lowest heat insulating capacity; it may adversely cause the indoor green house effect so that the indoor temperature is higher than the outside temperature. The heat-absorbing glass refers to the excessively dyed glass usually in brown or dark blue. Hence, developing intelligent window energy-saving technology and product is currently undergoing with all efforts in the U.S., Japan (Electrochromic Window Technology 2006; Smart Window Technology 2005; How Smart Window Work 2010) and some countries (Varthis \& Fotiadis 2006; Khalaj et al. 2007; Saraireh et al. 2007; Tee et al. 2010). However, Taiwan has dense population living in limited land; most people live or work in closed high-rise buildings that need tremendous air conditioning at the cost of excessive energy consumption in order to maintain a suitable living environment. A poor indoor air quality will significantly affects the health of the occupants; the common dizzy or lethargy problem often experienced by white-collar class is mainly caused by a high concentration of indoor carbon dioxide. Hence, the objective of this research is to develop an intelligent system to automatically adjust window opening to meet the requirements of maintaining a healthy and comfort indoor living environment while achieving energy savings. The system applies a wireless network of probes to detect multiple environmental factors that are related to the environmental quality, and a low-frequency electromagnetic wave to transmit the data to the control (Lin et al. 2011). Hence, the system will not interfere with the room design; it automatically detects the room environmental parameters to automatically adjust the window to achieve efficient control of the indoor environmental quality and to protect the health of the occupant.

\section{Design of an intelligent control system of automatic window}

The unique feature of this research is the development of a multiple, wireless detecting mechanisms to intelligently control the window automatically. As shown in figure 1, the system combines hardware, firmware, and software in an integrated design using the WSN sensor connected to the window opening control to form an automatic system that sends and receives signals for automatically adjusting the window openings in order to maintain the optimum indoor quality. The unique features of the system developed include: (i) using the standard protocol as the ZigBee transmission protocol to avoid the adverse effect of using electromagnetic wave on the long-term health of occupants, (ii) low power consumption due to power source sharing by 
(a)

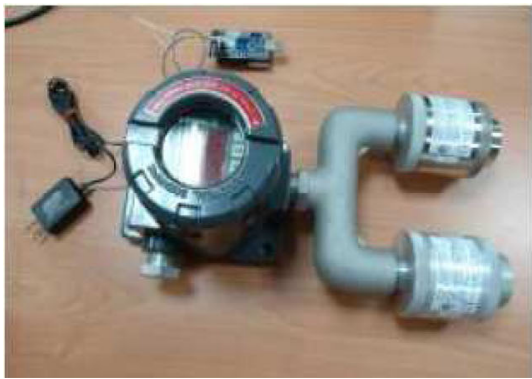

(b)

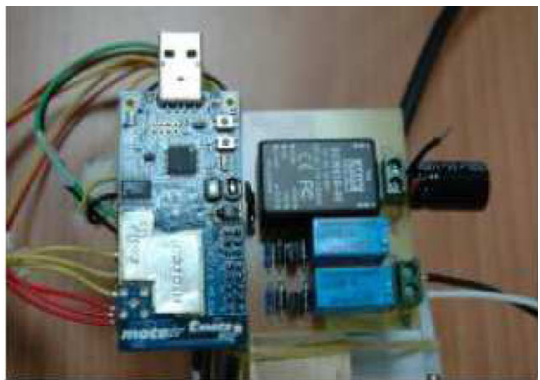

Figure 1. An intelligent control device of automatic window motor with diverse wireless sensor. (a) Smart control sensor for automatic window; (b) smart control panel for automatic window.

transmissions and sensors, (iii) diverse sensors that can be adjusted and integrated according to considerations on various environment and health factor, (iv) wireless random hop communication using the wireless mode of transmission to send signal sensor, so that the design is limited by the room space and design, (v) automatic trigger mechanism that is coped with the various control flow for adjusting the data dissemination for sensor threshold to start the connecting rod motor, (vi) expandable by using a embedded system language to develop the firmware and the data calculation for connecting the software and hardware based on the various required embedded system design.

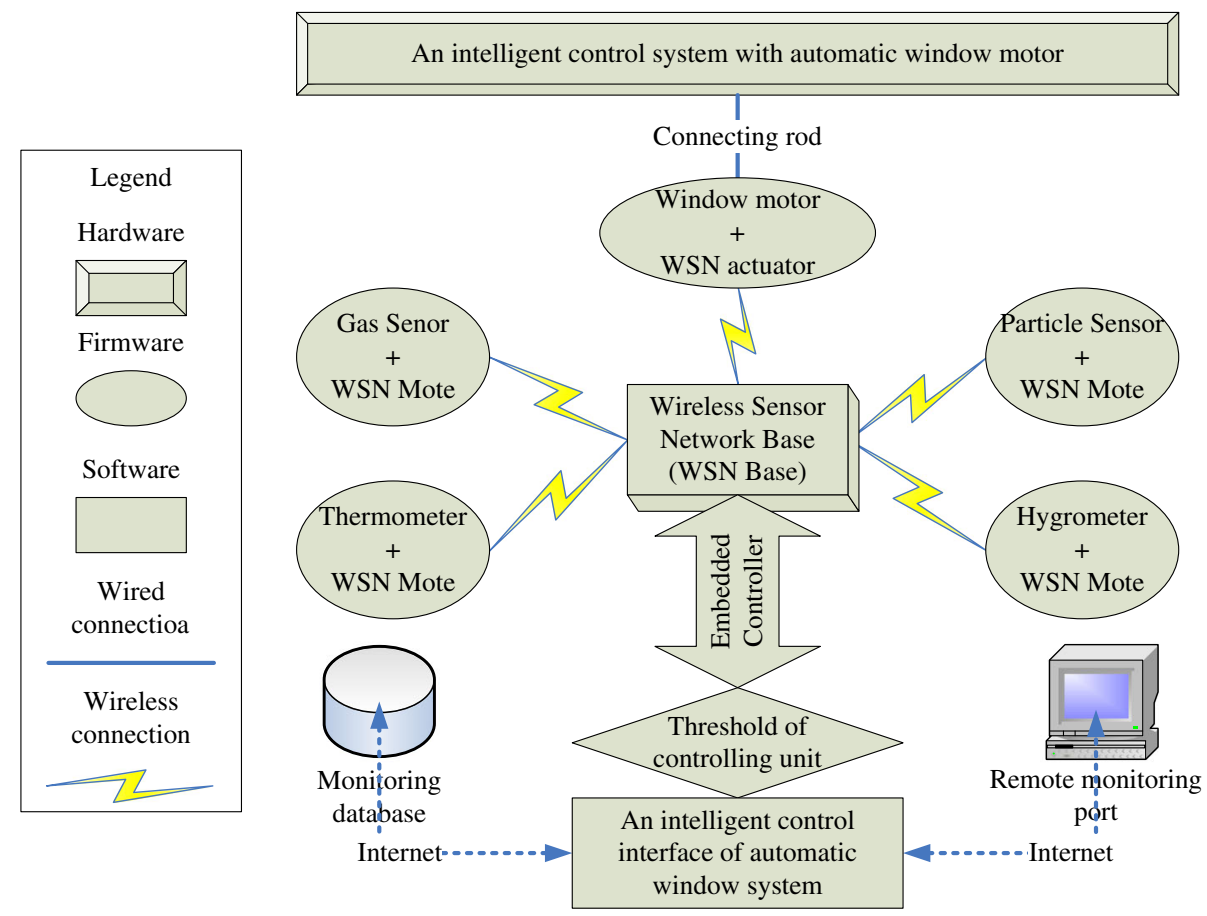

Figure 2. Network devices packet switching activities for an intelligent control system of automatic window motor with diverse wireless sensor. 
The major units used in this system include:

(i) The WSN motor control unit: the design that integrated WSN sensor and running motor equipment is capable of automatically accepting the wireless ZigBee signal for starting the motor to adjust the degree of window opening step-wise.

(ii) Deployment of multiple wireless sensor network: the improved WSN sensor is a wireless multiple system capable of monitoring multiple environmental factor that affect the indoor quality and the health of occupants.

(iii) WSN multiple monitoring information control system: an improved information receiving and sending unit that is capable of analysing and transmitting multiple information packet instantly. It is connected to the data bank of monitoring signal to form a threshold for forwarding appropriate signal to start the connecting rod of slide motor.

The automatic control system consists of three parts: hardware, firmware, and software. The composition and connection of these three parts are shown in figure 2 and illustrated in the following sections.

\subsection{Hardware design}

The hardware of the proposed system consists of: (i) intelligent window with a stretchable rod and a control motor, (ii) remodelled industrial sensors to monitor environmental conditions including temperature, humidity, concentration of carbon dioxide, particles and typical unhealthful gas, (iii) integrated system with wireless sensor network transmission (WSN Mote), improved WSN-enabled motor with an actuator unit, and a transmit-receive unit (WSN Base). Hardware information of the original industrial sensors is listed in table 1. In addition, a selfdesigned relay-circuit switch is combined with the WSN mote to connect various sensors so that environmental signals can be detected and sent by formatted packets that are compliant with the ZigBee protocol. Thus the WSN base can be controlled by a remote monitoring port through internet while the proposed system processes the monitoring database according to the thresholds.

\subsection{Firmware design}

The procedure of packet delivery is coded within the embedded system of the sensor and transmit-receive unit so that the diverse sensed signals can be sent to a variety of transmit-receive units through ad hoc network and multi hop transportation to activate the actuator motor. We

Table 1. Hardware information of environmental sensors for the automatic window system.

\begin{tabular}{|c|c|c|c|}
\hline Sensor & Model & Manufactory & Website \\
\hline Particle & PPD20V & Shinyei Co. & $\begin{array}{l}\text { http://www.shinyei.co.jp/STC/optical/ } \\
\text { dust_e.html }\end{array}$ \\
\hline Toxic gas & iTrans & Airmet Co. & $\begin{array}{l}\text { http://www.airmet.com.au/Product/ } \\
\text { The-iTrans-Gas-Detector.aspx }\end{array}$ \\
\hline Carbon dioxide & GD250W4E & Honeywell Co. & $\begin{array}{l}\text { http://customer-ap.honeywell.com/ } \\
\text { honeywell/ProductInfo.aspx/GD250W4E }\end{array}$ \\
\hline $\begin{array}{l}\text { Temperature/ } \\
\text { humidity }\end{array}$ & BATMote & $\begin{array}{l}\text { CeDInt research Center of } \\
\text { Technology University of Madrid }\end{array}$ & $\begin{array}{l}\text { http://batmote.cedint.upm.es/wiki/ } \\
\text { index.php/Main_Page }\end{array}$ \\
\hline
\end{tabular}


Table 2. Control command for automatic window motor.

\begin{tabular}{|c|c|c|}
\hline id & HEX String command & Motion \\
\hline 0 & 7E 44 0E 00000000000200890000 A6 62 7E & Motor Stop \\
\hline 1 & 7E 44 OF 00000000000200890001 CE AA 7E & Motor Start \\
\hline 2 & 7E $441000000000000200890002 \mathrm{~A} 3447 \mathrm{E}$ & Reverse Direction \\
\hline 3 & 7E 441100000000000200890003 CB 8C 7E & Min. Length (full open window) \\
\hline 4 & 7E 441200000000000200890004 D6 84 7E & Rod Position 1 \\
\hline 5 & 7E $441300000000000200890005 \mathrm{BE} 4 \mathrm{C} 7 \mathrm{E}$ & Rod Position 2 \\
\hline 6 & 7E $44140000000000020089000660557 \mathrm{E}$ & Rod Position 3 \\
\hline 7 & 7E 44150000000000020089000708 9D 7E & Max. Length (full close window) \\
\hline
\end{tabular}

followed the experience of previous study (Lin et al 2011) to determine the design procedure due to: (i) classifying the characteristics of sensor data, (ii) filtering available signals and parsing packet format as readable voltage data, (iii) converting the data into physical values such as concentration or degree, (iv) transporting sensed data to the system server at the back end, (v) controlling the window motor by HEX string command based on the criteria as shown in table 2. Thus, the actuator on the stretchable rod will be activated in a variety of motions, e.g., "stop," "start," "reverse," "minimum length," "maximum length," and three "rod position," after recognizing the command. Herein, the popular TinyOS embedded system is employed for the firmware design.

\subsection{Software design}

The interface developed between signal packets and embedded system is cooperated with the threshold of control criteria due to various environmental factors. The software enables automatic control while sending the command packet to the actuator of WSN motor to open or close

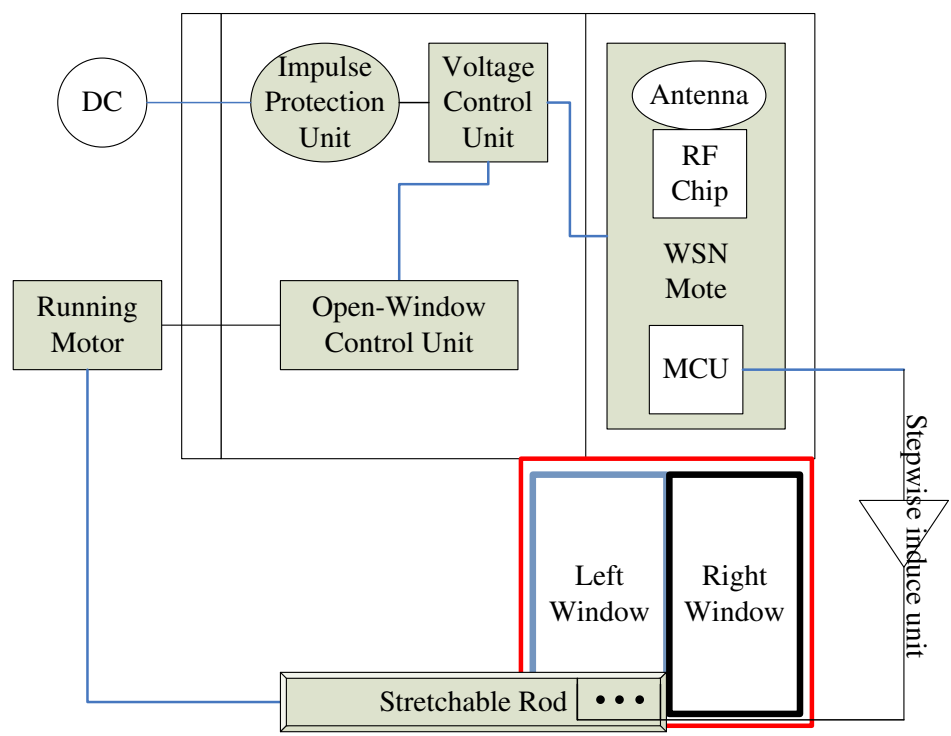

Figure 3. Improved WSN motor with actuator unit. 
the intelligent widow with step-wise function. Once the receiver of the system server obtains the sensed data, the analytical module of the backend system will begin to evaluate whether the detected data reach the threshold of the criteria. The computation will return the motion id as shown in table 2 and the relevant HEX string command is immediately sent to the actuator for running the motor of the stretchable rod. Additionally, this design provides surveillance database backup and remote monitoring interface to enhance the control criteria due to adjustable thresholds. Figure 3 shows the improved motor with actuator unit that integrates the WSN mote and the actuator motor. Due to the block diagram of the control system, the direct circuit (DC) supplies power to start up the running motor for moving the rod of the left window.

Here, the components of the block diagram are defined. (i) Impulse protection unit-the circuit contains the surge protector to avoid impulse noises disturbing the control unit. (ii) Voltage control unit - the circuit contains the voltage regulator to ensure stable voltage input of the WSN mote. (iii) Open-Window control unit - the program provides intelligent control commands upon thresholds to drive the motor. (iv) WSN mote-the mote complies with the protocols of WSN sensors and transact the WSN data with the server side. (v) Running motor-the motor obtains and runs the control command to move the stretchable rod. Thus, the prototype of software system is established by open source Java technology while the real-time environmental monitoring function with model-view-controller design pattern (Lin et al 2012) is considered.

\section{System evaluation, test results and discussions}

Two shelters made of the phenolic resin board (figure 4) are exposed to direct sunshine in Central Taiwan, an island surrounded by sea, is located in subtropical region. Thermal sensors are placed on the interior and exterior sides for recording changes of temperatures. In August 2010, the interior and outside temperatures are recorded at different hours for every day with the shelters

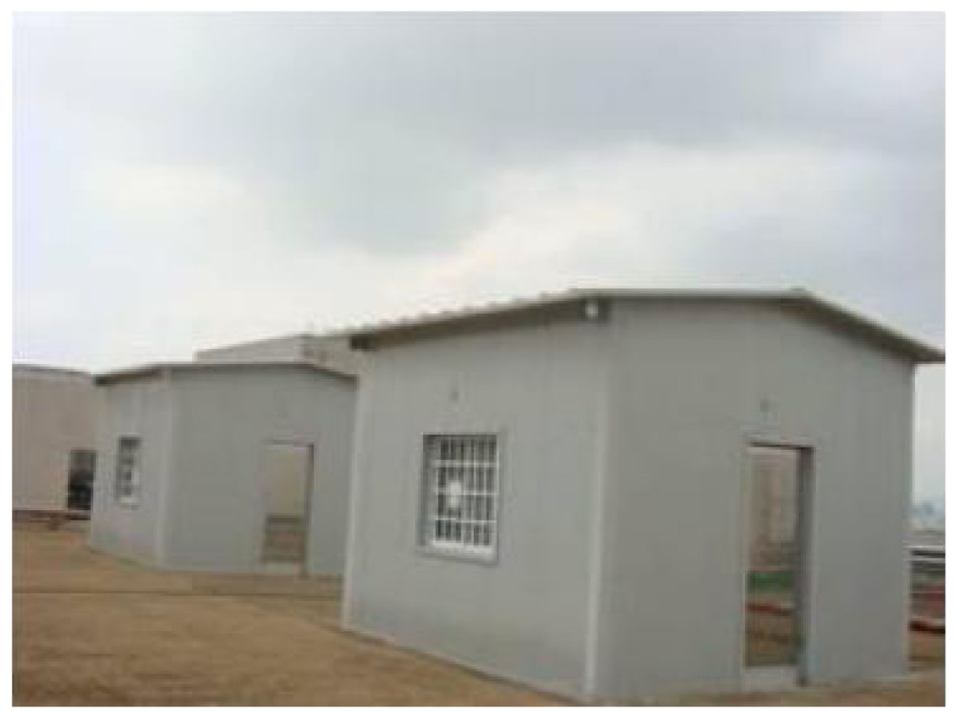

Figure 4. The experimental shelter used in this experiment. 


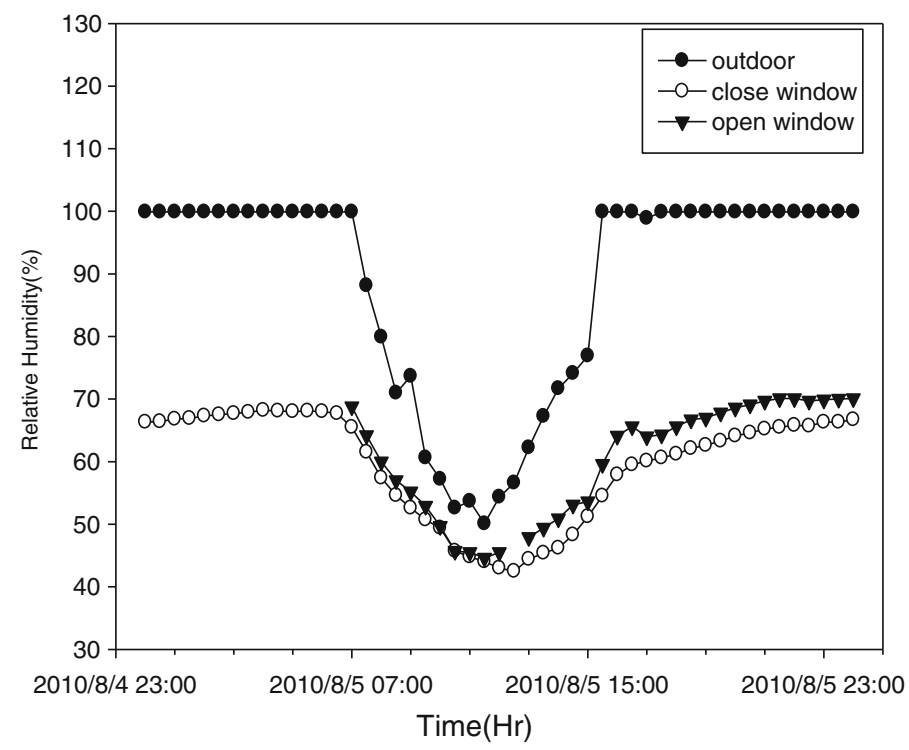

Figure 5. The comparison of relative humidity of outdoor and two experimental shelters.

door and windows closed or opened. One of shelters is installed this purposed intelligent control system of automatic window. This device will be automatically started based on the indoor temperature, humidity and carbon dioxide consistency.

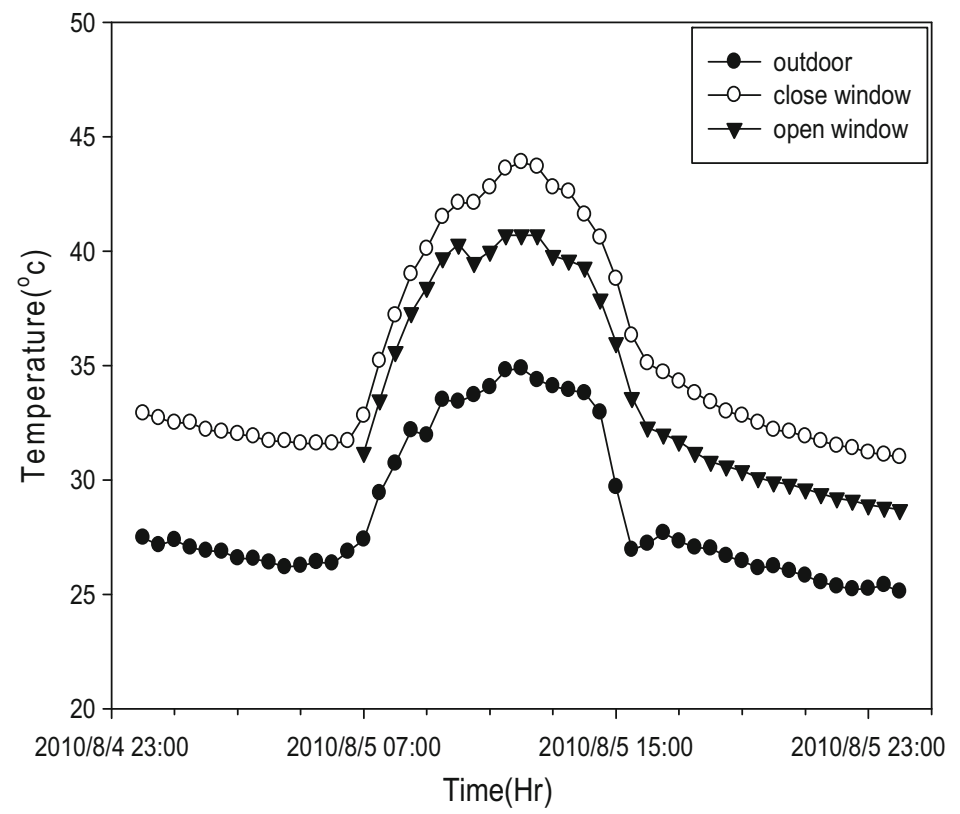

Figure 6. The comparison of temperature of outdoor and two experimental shelters. 


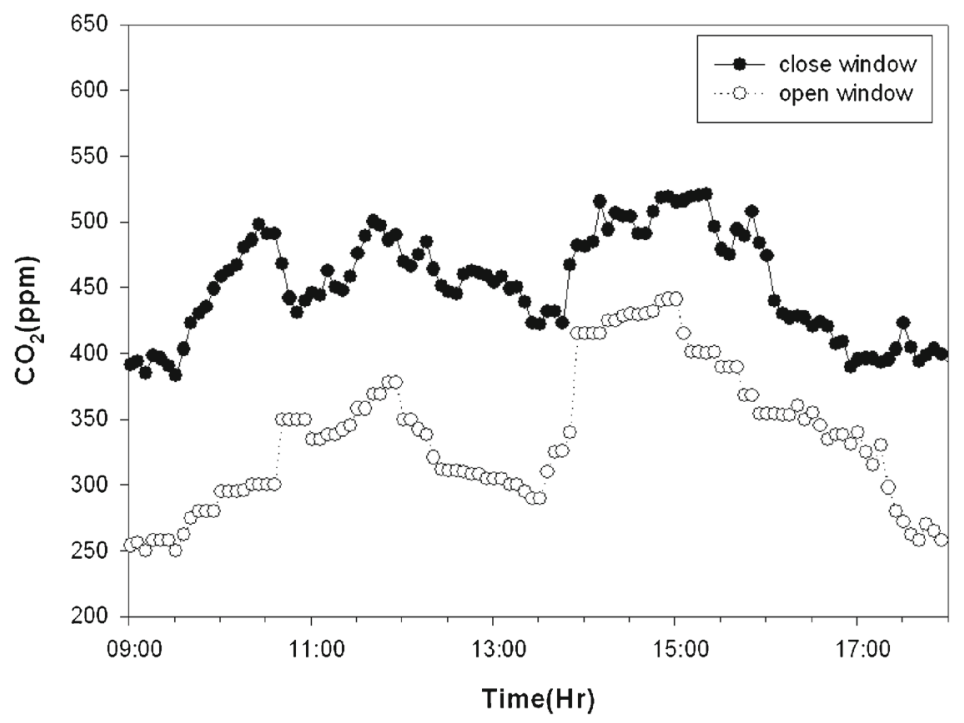

Figure 7. The comparison of carbon dioxide of two experimental shelters—close window and open window.

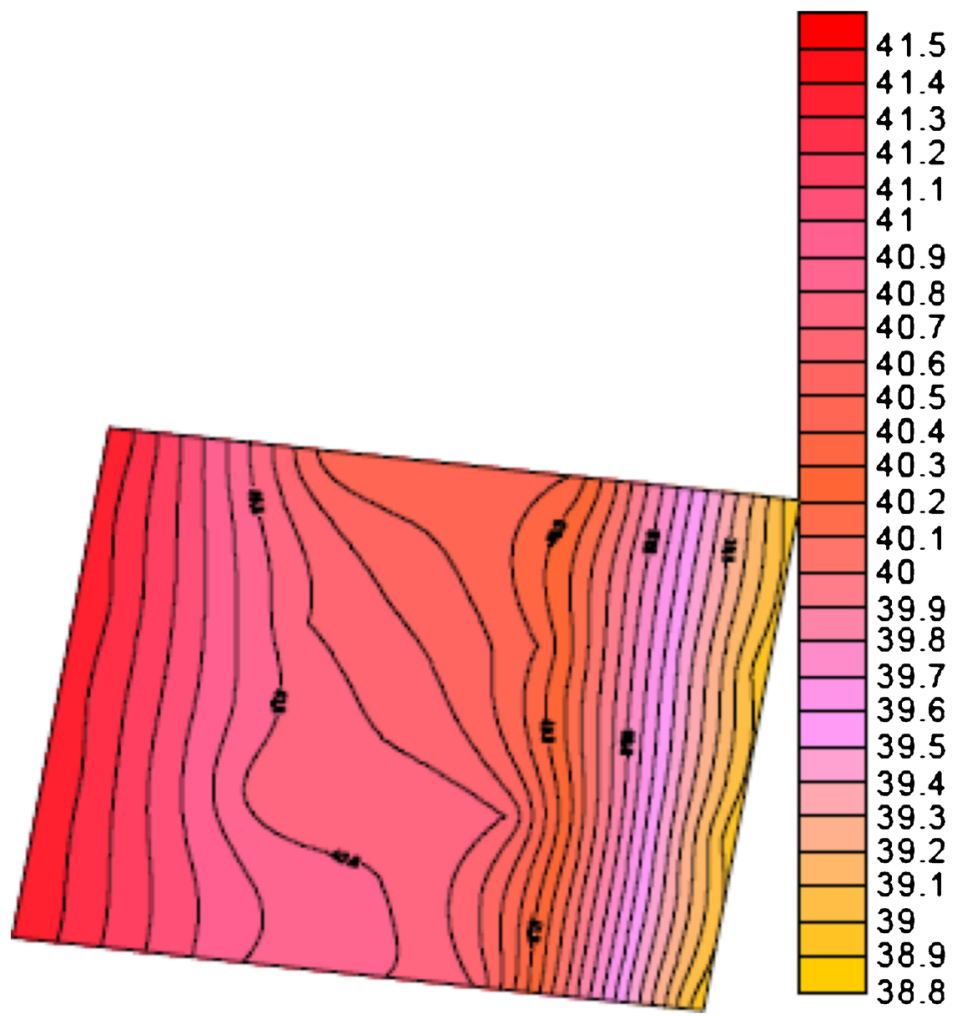

Figure 8. Variations of indoor spacious temperatures of experimental shelter with close window. 
Results of laboratory studies show that when the outside relative humidity is a near $100 \%$ with the outside temperature higher than indoor temperature, the system is not activated so that the outside humidity and temperature cannot adversely affect the indoor comfort. At around 6 a.m., the outside humidity begins to decrease and the indoor temperature starts to elevate, the system automatically issues an instruction to open the window. The indoor humidity remains almost the same before and after the window is opened (figure 5). However, the difference between outside and indoor temperatures increases with time (figure 6). At noon, the window is fully open; the maximum difference between indoor temperature for room with open window and the room with closed window is observed. This observation demonstrates that opening the window will lower the indoor room temperature. Additionally, the room with open window has lower concentration of carbon dioxide than the room with closed window (figure 7). Thus, the benefit of the proposed system can be realized.

Variations of indoor spacious temperatures as monitored using thermal sensors show that the temperature near the window that faces the sun is $2^{\circ} \mathrm{C}$ higher for the room with closed window than for the room with open window. The temperature difference is $3-4^{\circ} \mathrm{C}$ for the wall on the opposite direction of the sun. The distribution field of room temperature reveals that the solar radiation heat cannot be properly dissipated in the room with the window closed; it accumulated to raise the room temperature as shown in figures 8 and 9, respectively. With the window opened, the heat gradually dissipated through the open window so that the room temperature decreases to a comfort level.

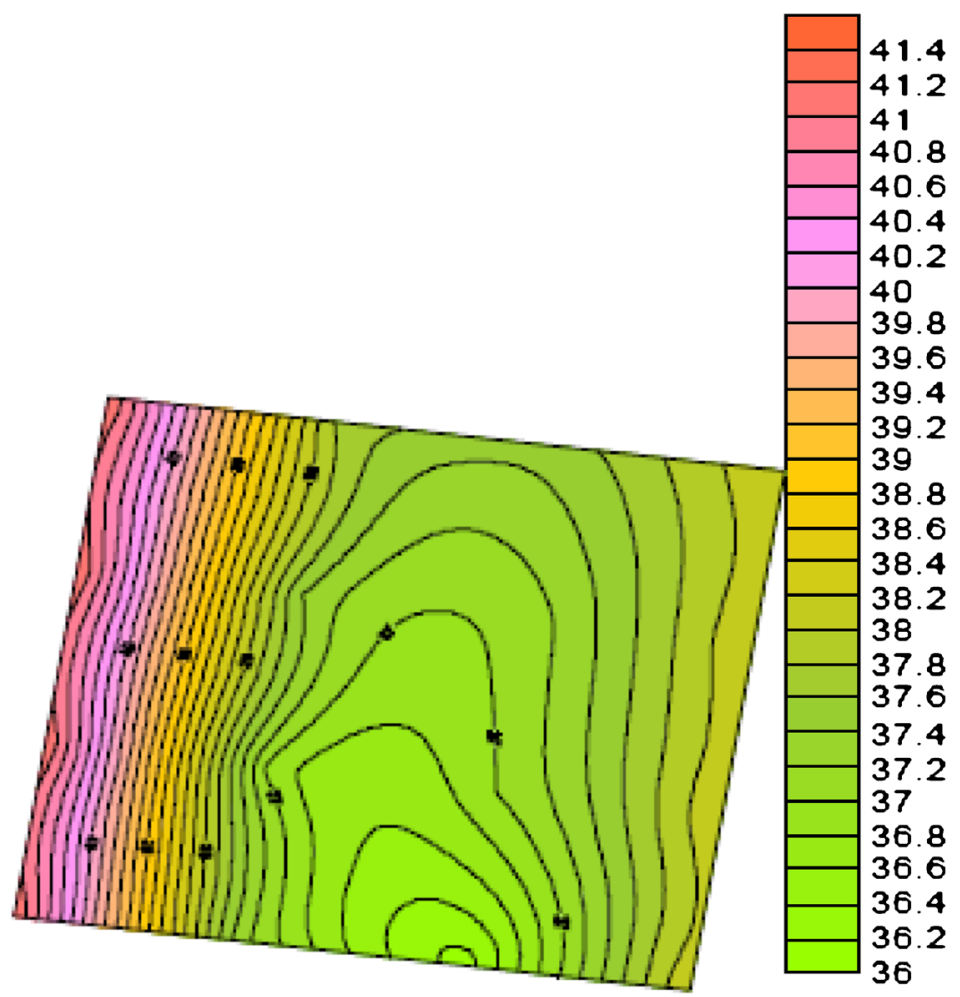

Figure 9. Variations of indoor spacious temperatures of experimental shelter with open window. 


\section{Conclusions}

The concept of combining intelligent technology and automatic control is implemented in this research to use the improved sensing device for controlling the living environment by adjusting window openings. Laboratory studies show the following results:

(i) The WSN technology, which transmits signals using low frequencies with low power consumption, can be used to control the environmental factors for achieving energy savings without causing health hazards to the occupants.

(ii) Developing an embedded system software to interface the software and hardware will add more flexibility and expandability of the control system in the overall application integration.

(iii) The design of an automatic control system to activate the intelligent window with connecting rod motor unit is effective in achieving the objective of controlling the window opening automatically and intelligently.

(iv) The slide motor can be designed to fit windows with various dimensions as requested by customers.

The concepts of green energy environment and environmental health risks have been incorporated in the design of the proposed system to instantaneously adjust the room air flow and circulation for improving indoor environment quality which is based on the environmental monitoring sensors.

\section{Acknowledgement}

The National Science Council of Taiwan has supported this research through grant No. NSC 98-3114-E-167-001 and NSC 100-3113-E-167-001.

\section{References}

Advancment Electrochromic Window 2006 Information on http://windows.lbl.gov/comm_perf/Electrochromic/ ec_tech.html

How Smart Window Work 2010 Information on http://tlc.howstuffworks.com/home/smart-window4.htm

Khalaj A, Yazdani N and Rahgozar M 2007 Effect of the contention window size on performance and fairness of the IEEE 802.11 standard. Wirel. Pers. Commun. 43: 1267-1278

Lin H C, Kan Y C and Hong Y-M 2011 The comprehensive gateway model for diverse environmental monitoring upon wireless sensor network. IEEE Sensors J. 11(5): 1293-1303

Lin H C, Hong Y-M and Kan Y-C 2012 The backend design of an environmental monitoring system upon real-time prediction of groundwater level fluctuation under the hillslope. Environ. Monit Assess. 184(1): 381-395

Saraireh M, Saatchi R, Al-Khayatt A and Strachan R 2007 Assessment and improvement of quality of service in wireless networks using fuzzy and hybrid genetic-fuzzy approaches. Artif. Int. Rev. 27: 95-111

Smart Window Technology 2005 Information on http://statusreports.atp.nist.gov/reports/92-01-0123TEXT. html

Tee Z H, Ang L M and Seng K P 2010 Smart guide system to assist visually impaired people in an indoor environment. IETE Tech. Rev. 27: 455-464

Varthis E G and Fotiadis D I 2006 A comparison of stop-and-wait and go-back-N ARQ schemes for IEEE 802.11e wireless infrared networks. Comput. Commun. 29: 1015-1025 